PROCEEDINGS OF THE

AMERICAN MATHEMATICAL SOCIETY

Volume 131, Number 7, Pages 2223-2233

S 0002-9939(02)06772-2

Article electronically published on October 15, 2002

\title{
THE RATIONAL LS-CATEGORY OF $k$-TRIVIAL FIBRATIONS
}

\author{
MAXENCE CUVILLIEZ AND BARRY JESSUP
}

(Communicated by Ralph Cohen)

\begin{abstract}
We provide new upper and lower bounds for the rational LScategory of a rational fibration $\xi: F \rightarrow E \rightarrow K(\mathbf{Q}, 2 n)$ of simply connected spaces that depend on a measure of the triviality of $\xi$ which is strictly finer than the vanishing of the higher holonomy actions. In particular, we prove that if $\xi$ is $k$-trivial for some $k \geq 0$ and $H^{*}(F)$ enjoys Poincaré duality, then cat $_{0} E \geq \operatorname{cat}_{0} F+k$.
\end{abstract}

\section{§1. INTRODUCTION}

The Lusternik-Schnirelmann category of a space $S$, denoted cat $S$, is the least number of open sets, less one, which cover $S$ and are contractible in $S$. It is a subtle measure of the complexity of $S$ which is difficult to compute except where it agrees with other well-known homotopy invariants, such as $\operatorname{dim} S$ or the cup length in the cohomology ring. If $S$ is simply connected and has the homotopy type of a CW complex of finite type, the rational category of $S$, $\operatorname{cat}_{0} S:=\operatorname{cat}_{\mathbf{Q}}$, introduced by Berstein, is a lower bound for cat $S$ that is more amenable to computation because Felix and Halperin [FH] provided a complete algebraic description of $\operatorname{cat}_{0} S$ in terms of a Sullivan minimal model of $S$.

We now know that $\operatorname{cat}_{0}\left(S_{1} \times S_{2}\right)=\operatorname{cat}_{0} S_{1}+\operatorname{cat}_{0} S S_{1}^{1}$ [FHL], so a natural question is to find conditions on a non-trivial fibration $\xi: F \rightarrow E \rightarrow B$ which permit estimates of $\mathrm{cat}_{0} E$ in terms of data associated to the "twisting". Some recent upper bounds in this spirit are provided in [CFJP, [JS] and [C2]. Lower bounds include the Mapping Theorem [FH], which in particular guarantees that $\operatorname{cat}_{0} E \geq \operatorname{cat}_{0} F$ when $F \hookrightarrow E$ induces an injection in rational homotopy. Extending this, various estimates of the form cat $_{0} E \geq$ cat $_{0} F+k$ for $k>0$ have been obtained using additional hypotheses on either the holonomy action and $B[\mathrm{~J}]$, on the homotopy Lie algebra of $E$ [GJ], or on the higher holonomy actions of $\xi$ itself [C1], [C2].

In this paper we attempt to unify and extend some of these ideas by introducing a new measure of the triviality of $\xi$ which is strictly finer than the vanishing of the higher holonomy operations.

Received by the editors October 10, 2000 and, in revised form, February 21, 2002.

2000 Mathematics Subject Classification. Primary 53C29, 55M30, 55P62, 55R05.

Key words and phrases. Lusternik-Schnirelmann category, holonomy, minimal model.

This research was partially supported by L'Université Catholique de Louvain-la-Neuve and by the National Science and Engineering Research Council of Canada. The second author thanks colleagues at UCL for their unstinting hospitality during a recent visit.

${ }^{1} \mathrm{~A}$ fact not true for cat. See [I]. 
To describe this new measure of triviality, let $G_{k} E \rightarrow E$ denote the $k^{\text {th }}$ Ganea fibration over $E$, and let $F \bowtie_{E} G_{k} E$ be the join of $F$ and $G_{k} E$ over $E$, with induced map $F \stackrel{j_{k}}{\longrightarrow} F \bowtie_{E} G_{k} E$. (Recall that the join $X \bowtie_{Y} Z$ of $X \rightarrow Y \leftarrow Z$ may be obtained by a pull-back followed by a push-out.) Then, we make the following

Definition. The fibration $\xi$ is trivial of order $k$ (or $k$-trivial) if $j_{k}$ has a homotopy retraction, i.e., there exists a map $r$ in the diagram

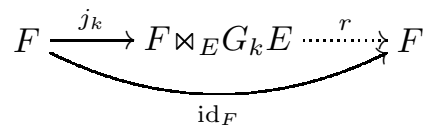

making it commute up to homotopy.

Since the map $j_{k}$ factorizes through $j_{k-1}, k$-trivial fibrations are also $(k-1)$ trivial. Moreover, a trivial fibration in the usual sense is trivial of arbitrarily high order.

In rational homotopy, 0-triviality of the fibration is exactly the (single) hypothesis of the Mapping Theorem. When $k=1$, it is precisely the first hypothesis of GJ, Thm. 1] in that particular case.

The vanishing of the $k^{\text {th }}$ (higher) holonomy action [C2] implies, but is not equivalent to, the $k$-triviality of the fibration. To see this, we recall that the $k^{\text {th }}$ holonomy action of $F \hookrightarrow E \rightarrow B$ vanishes iff

$$
i: F \rightarrow G_{k+1}(E, F)
$$

has a homotopy retraction (where $G_{k}(E, F)$ is the homotopy pullback of $E \rightarrow B$ and $G_{k} B \rightarrow B$ ), and that there is a natural map $F \bowtie_{E} G_{k} E \rightarrow G_{k+1}(E, F)$ which is compatible with $i$ and $j_{k}$.

Recall the definition of relative LS-category in the sense of Fadell and Husseini $[\mathrm{FaHu}]$. If $(M, A)$ is an NDR pair, the relative category of $(M, A)$, denoted $\operatorname{cat}(M, A)$, is the least integer $n$ such that there exists an open covering $\left(M_{j}\right)_{0 \leq j \leq n}$ of $M$ such that $M_{0} \hookrightarrow M$ factorizes up to homotopy (relative to $A$ ) through $A$ and that the open sets $M_{j}, j \geq 1$, are contractible in $M$. Moreover, $\operatorname{cat}(M, A) \leq n+1$ iff the map $i \bowtie g_{n}: A \bowtie_{M} G_{n} M \rightarrow M$ has a homotopy section $s$ satisfying $s \circ i \simeq j_{k}$, where $j_{k}$ is the inclusion $A \hookrightarrow A \bowtie_{M} G_{k} M[\mathrm{M}]$. Note that we have

$$
\text { cat } M / A \leq \operatorname{cat}(M, A) \leq \operatorname{cat} M+1 \text {. }
$$

Remark 1. Note that when $\operatorname{cat}(E, F) \leq k+1$ and the fibration $\xi: F \hookrightarrow E \rightarrow B$ is $k$-trivial, then $\xi$ is in fact homotopically trivial, since the composition of the two homotopy retractions guaranteed by these conditions shows that $F \hookrightarrow E$ has a homotopy retraction. This has two interesting consequences:

- If $\xi$ is a $k$-trivial fibration which is not homotopically trivial, then $\operatorname{cat}(E, F)>$ $k+1$ and, in general, for any $k$-trivial fibration, we have

$$
\operatorname{cat}(E, F)>\min (\operatorname{cat} B-1, k+1) \text {. }
$$

Indeed, assume $\operatorname{cat}(E, F) \leq k+1$. Then the fibration $\xi$ is homotopically trivial and

$$
\operatorname{cat}(E, F)=\operatorname{cat}(B \times F, F) \geq \operatorname{cat} B .
$$

- Since $\operatorname{cat}(E, F) \leq$ cat $E+1$, if $\xi$ is a $k$-trivial fibration with cat $E \leq k$, then the fibration is homotopically trivial in the usual sense. 
With $K(\mathbf{Q}, 2 n)$ denoting as usual an Eilenberg-Mac Lane space and $\operatorname{cat}_{0}(E, F)$ denoting $\operatorname{cat}\left(E_{\mathbf{Q}}, F_{\mathbf{Q}}\right)$, we can now state

Theorem 1. Suppose $F \rightarrow E \rightarrow K(\mathbf{Q}, 2 n)$ is a rational $k$-trivial fibration for some $k \geq 0$. Then:

- cat $_{0} E \leq \operatorname{cat}_{0}(E, F)+\max \left(\operatorname{cat}_{0} F, k+1\right)-k-1$.

- If in addition $H^{*}(F ; \mathbf{Q})$ satisfies Poincaré duality, we have $\operatorname{cat}_{0} E \geq \operatorname{cat}_{0} F+k$.

In the general case, we have cat $M \leq \operatorname{cat}(M, A)+\operatorname{cat} A$ and so the first inequality is an improvement on this bound. Similary, the second point of Remark 1 implies that cat $E>k$ and the second inequality is an improvement on this bound.

Stated this way, the second inequality relies on the equality cat $t_{0}=e_{0}$ for Poincaré duality spaces [FHL]. Here, $e_{0}$ denotes Toomer's invariant $[\mathrm{T}$, which is the largest $p$ such that in the spectral sequence of Milnor and Moore, $E_{\infty}^{p, \star} \neq 0$. Under our assumptions, $H^{*}(E ; \mathbf{Q})$ is also a P.d.a [FHT2, Thms. $\left.4.3 \& 3.1\right]$, so what we actually prove is that $\mathrm{e}_{0} E \geq \mathrm{e}_{0} F+k$. As already mentioned, second point of Theorem 1 is a generalization of [GJ, Thm. 1], and, once we have algebraically characterized the condition of $k$-triviality, it is proved in the same way.

Theorem 1 is proven using the standard methods of rational homotopy, and we remark that it is a straightforward matter to check for $k$-triviality when one has a minimal model of $E$, especially when $E$ is an elliptic space, i.e., when $\pi_{*} E \otimes \mathbf{Q}$ and $H^{*}(E ; \mathbf{Q})$ are finite dimensional.

This paper is organized as follows. In the next section, we give a characterization of our hypotheses at the level of minimal models, and we state a proposition that will imply the second point of Theorem 1 . In section 3 , we will prove the two points of Theorem 1. In the final section, we present 3 examples to illustrate our results.

\section{$\S 2$. RATional Homotopy and cat $_{0}$}

All our spaces will be simply connected with the homotopy type of CW complexes with rational cohomology of finite type. We will work with $\mathbf{Q}$ as ground field and our principal tools are Sullivan models. A detailed description of these and the standard tools of rational homotopy can be found in [FHT1]. For our purposes, we recall the following.

Sullivan [S] defined a contravariant functor $\mathcal{A}_{P L}$ which associates to each space $S$ a commutative graded differential algebra (hereafter cgda) $\mathcal{A}_{P L}(S)$ which represents the rational homotopy type of $S$. He also constructed, for each simply connected cgda $(A, d)$ (i.e. satisfying $\left.H^{0}(A, d)=H^{1}(A, d)=0\right)$, another cgda $(\Lambda X, d)$ and a map

$$
(\Lambda X, d) \stackrel{\simeq}{\rightarrow}(A, d)
$$

which induces an isomorphism in cohomology (hereafter called a quasi-isomorphism), where $\Lambda X$ denotes the free commutative-graded algebra on the graded vector space $X=\sum_{n \geq 2} X^{n}$, which has a well ordered, homogeneous basis $\left\{x_{\alpha}\right\}$ such that, if $X_{<\alpha}$ denotes $\operatorname{span}\left\{x_{\beta} \mid \beta<\alpha\right\}$, we have $d x_{\alpha} \in \Lambda^{\geq 2}\left(X_{<\alpha}\right)$. The cgda $(\Lambda X, d)$ is called a (minimal) Sullivan model of $(A, d)$ or a Sullivan model of $S$ if $(A, d)=\mathcal{A}_{P L}(S)$.

He also defined a geometric realization functor |.| which converts a (minimal) Sullivan model $(\Lambda X, d)$ (of finite type) into a rational space $|(\Lambda X, d)|$ so that $(\Lambda X, d)$ 
is a Sullivan model for $|(\Lambda X, d)|$. These functors define bijections

$$
\begin{aligned}
& \left\{\begin{array}{c}
\text { rational homotopy } \\
\text { types of spaces }
\end{array}\right\} \longleftrightarrow\left\{\begin{array}{c}
\text { isomorphism classes of } \\
\text { minimal Sullivan models }
\end{array}\right\}, \\
& \left\{\begin{array}{c}
\text { homotopy classes of } \\
\text { maps between rational spaces }
\end{array}\right\} \longleftrightarrow\left\{\begin{array}{c}
\text { homotopy classes of maps } \\
\text { between minimal Sullivan models }
\end{array}\right\} \text {. }
\end{aligned}
$$

When two cgda's have isomorphic minimal models, we say that they are quasiisomorphic cgda's, even though there may not be a quasi-isomorphism between them.

Let $\Lambda(t, d t)$ be the cgda generated as an algebra by $t$ in degree 0 , and $d t$ in degree 1 , with $d(t)=d t$ and $d(d t)=0$, and let $\varepsilon_{0}, \varepsilon_{1}: \Lambda(t, d t) \rightarrow(\mathbf{Q}, 0)$ denote the unique maps satisfying $\varepsilon_{i}(t)=i t$. If $(\Lambda X, d)$ is a minimal model and $\left(A, d_{A}\right)$ is any 0 -connected cgda, two maps $\phi_{0}, \phi_{1}:(\Lambda X, d) \rightarrow\left(A, d_{A}\right)$ are homotopic if there is a map

$$
\Phi:(\Lambda X, d) \rightarrow\left(A, d_{A}\right) \otimes \Lambda(t, d t)
$$

with $\phi_{i}=\varepsilon_{i} \Phi, i=0,1$.

If $\phi:(A, d) \rightarrow(B, d)$ is a morphism of 1-connected cgda's, a Sullivan or relative model of $\phi$ is a factoring $\phi=\psi i$ in

$$
\left(A, d_{A}\right) \stackrel{i}{\rightarrow}(A \otimes \Lambda X, d) \stackrel{\psi}{\underset{\simeq}{\longrightarrow}}\left(B, d_{B}\right)
$$

where $i(a)=a \otimes 1$ for $a \in A, \psi$ is a quasi-isomorphism and

$$
(\Lambda X, \bar{d}):=(A \otimes \Lambda X, d) /\left(A^{+} \otimes \Lambda X, d\right)
$$

is a minimal Sullivan model.

For every Serre fibration $\xi: F \stackrel{i}{\rightarrow} E \stackrel{p}{\rightarrow} B$ of simply connected spaces, there is a commutative diagram of augmented cgda's

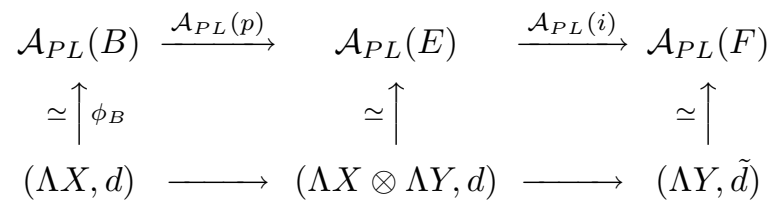

in which $(\Lambda X, d)$ and $(\Lambda Y, \bar{d})$ are Sullivan models for $B$ and $F$ respectively, and the bottom row is the Sullivan model of $\mathcal{A}_{P L}(p) \circ \phi_{B}$. The bottom row of this diagram is called a minimal K-S extension and a minimal model of the fibration. In general, the middle cgda need not be a minimal model of $E$, but will be precisely when the kernel of the homomorphism $\pi_{k}(F) \rightarrow \pi_{k}(E)$ is strictly torsion.

Now let $S$ be a space and $(\Lambda X, d)$ a minimal model of $S$. The projection $\Lambda X \rightarrow \Lambda X / \Lambda^{>m} X$ induces a differential $D$ in $\Lambda X / \Lambda^{>m} X$ which makes it a map of differential algebras. Let

$$
(\Lambda X, d) \rightarrow(\Lambda X \otimes \Lambda V, d) \stackrel{\simeq}{\longrightarrow}\left(\Lambda X / \Lambda^{>m} X, D\right)
$$

be a relative model for this projection. The rational category of $(\Lambda X, d)$, denoted cat $_{0} S$, is the least $m$ such that there is a map $r:(\Lambda X \otimes \Lambda V, d) \rightarrow(\Lambda X, d)$ of cgda's which satisfies $r(x)=x$ for all $x \in X$. Félix and Halperin [FH] proved that $\operatorname{cat}_{0} S=\operatorname{cat}_{\mathbf{Q}}$. 
Toomer's invariant, denoted $\mathrm{e}_{0} S$, may also be defined as the least $m$ for which there is a map $r:(\Lambda X \otimes \Lambda V, d) \rightarrow(\Lambda X, d)$ of graded differential vector spaces which satisfies $r(x)=x$ for all $x \in X$. Clearly, $e_{0} S \leq \operatorname{cat}_{0} S$ and it is straightforward that

$$
e_{0} S=\sup \left\{k \mid \exists \alpha \in \Lambda^{\geq k} X \text { with } 0 \neq[\alpha] \in H^{*}(S ; \mathbf{Q})\right\} .
$$

Moreover, if $S$ is a Poincaré duality space, the top class is the 'longest' class, that is, we may assume there exists $\alpha \in \Lambda^{\geq e_{0} S} X$ such that $[\alpha] \neq 0$ and $H^{>|\alpha|}(S ; \mathbf{Q})=0$.

We are now in a position to interpret the $k$-triviality of the fibration on the level of minimal models. Let $E$ and $F$ be simply connected rational spaces and $\xi: F \rightarrow E \rightarrow K(Q, 2 n)$ a rational fibration. Suppose $k \geq 0$, that $g_{k}: G_{k} E \rightarrow$ $E$ is the $k^{t h}$ Ganea fibration of $E$ and let $F \bowtie_{E} G_{k} E$ be the join of $g_{k}$ and the inclusion $i: F \rightarrow E$. That is, if $P$ denotes the homotopy pullback of $g_{k}$ and $i$, then $F \bowtie_{E} G_{k} E$ is the homotopy pushout of the projection $P \rightarrow F$ and $P \rightarrow G_{k} E$. Let $i \bowtie g_{k}: F \bowtie_{E} G_{k} E \rightarrow E$ be the induced map as in the diagram

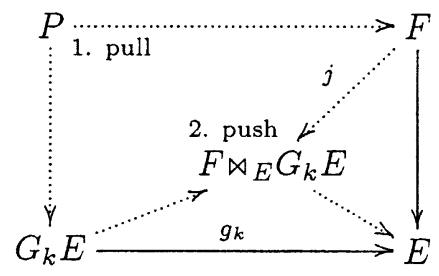

where we have denoted by $j$ the inclusion $F \rightarrow F \bowtie_{E} G_{k} E$.

Proposition 2. The fibration $\xi$ is $k$-trivial iff there exists a minimal model

$$
\Lambda(a ; 0) \rightarrow \Lambda(a, X ; d) \rightarrow \Lambda(X ; \bar{d})
$$

of $\xi$ with

$$
d: X \rightarrow \Lambda X \oplus \Lambda^{+} a \cdot \Lambda^{>k}(a, X) .
$$

We remark [C2 that the vanishing of the $k^{\text {th }}$-higher holonomy is equivalent, on this level, to the existence of a model of $\xi$ as above with

$$
d: X \rightarrow \Lambda X \oplus \Lambda^{>k+1} a \cdot \Lambda(a, X),
$$

and an example exhibiting that this is stronger than the above is presented in the last section.

The proof of Proposition 2 will follow directly from Lemmas 3-6. However, before proceeding with them, we indicate why 1-triviality of the fibration is equivalent to the hypotheses of [GJ. Theorem 1].

If $(\Lambda X, d)$ is the minimal model of $S$, then as graded vector spaces, $L_{S}^{n}:=$ $\operatorname{Hom}\left(X^{n}, \mathbf{Q}\right) \cong \pi_{n}(S) \otimes \mathbf{Q}$. Then, $L_{S}^{n} \cong \pi_{n}(\Omega S) \otimes \mathbf{Q}$, and the homotopy Lie algebra of $S_{\mathrm{Q}}$ is encoded in the minimal model $(\Lambda X, d)$ as follows.

The differential $d$ can be written as a sum of derivations $d=d_{2}+d_{3}+\ldots$ where $d_{i}: X \rightarrow \Lambda^{i} X$. The fact that $d^{2}=0$ implies the same for $d_{2}$. The dual of $d_{2}: X \rightarrow \Lambda^{2} X$ induces a bilinear and antisymmetric [,] : $L_{S} \otimes L_{S} \rightarrow L_{S}$, which represents the Samelson product in $\pi(\Omega S) \otimes \mathbf{Q}$. The Jacobi identity for [,] is equivalent to $d_{2}^{2}=0$. One calls $\left(L_{S},[],\right)$ the rational homotopy Lie algebra of $S$.

In particular, if $\left\{x_{\alpha}\right\}$ is a K-S basis for $X$, and $\left\{\hat{x}_{\alpha}\right\}$ is its dual basis, then

1. $\left[\hat{x}_{\alpha}, \hat{x}_{\beta}\right]=0$ iff the coefficient of $x_{\alpha} x_{\beta}$ in $d_{2} x_{\gamma}$ is zero for all $\gamma$, so that 
2. $\hat{x}_{\alpha}$ is in the centre of $L_{S}$ iff

$$
d_{2}: X \rightarrow \Lambda^{2}\left\langle x_{\beta} \mid \beta \neq \alpha\right\rangle \text {. }
$$

When no confusion will arise, we shall say that $x_{\alpha}$ belongs to the centre of $L_{S}$ if $\hat{x}_{\alpha}$ does.

Thus, a rational fibration $F \hookrightarrow E \rightarrow K(\mathbf{Q}, 2 n)$ is $k$-trivial iff the fundamental homotopy class of the base, when viewed in $L_{E}$, is in the center of $L_{E}$.

We can now proceed with the proof of Proposition 2.

Lemma 3. Let

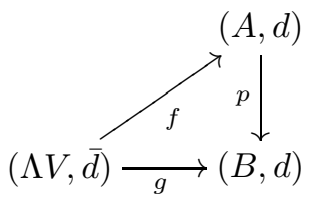

be a commutative diagram with $p$ surjective, and suppose that $g^{\prime}$ is an another map homotopic to $g$. Then there exists a map $f^{\prime}:(\Lambda V, \bar{d}) \rightarrow(A, d)$, homotopic to $f$ satisfying $p \circ f^{\prime}=g^{\prime}$.

The proof of this lemma is a standard lifting argument.

Lemma 4. Let $\Lambda(a ; 0) \rightarrow \Lambda(a, X ; d) \stackrel{p}{\rightarrow} \Lambda(X ; \bar{d})$ be any minimal model of $\xi$ and suppose that $W_{k}$ is a geometric realization of $\left(\Lambda(a, X) / \Lambda^{>k}(a, X), D\right)$. Then,

$$
\left(\Lambda(a, X) / a \cdot \Lambda^{>k}(a, X), D\right)
$$

is a model of $F \bowtie_{E} W_{k}$.

Proof. Consider a relative model of $(\Lambda(a, X), d) \stackrel{q}{\rightarrow}\left(\Lambda(a, X) / \Lambda^{>k}(a, X), D\right)$

$$
\begin{array}{r}
(\Lambda(a, X), d) \stackrel{q}{\longrightarrow}\left(\Lambda(a, X) / \Lambda^{>k}(a, X), D\right) \\
\left.\simeq\right|^{\uparrow} \\
(a, X) \otimes \Lambda Y, \delta)
\end{array}
$$

and let $(A, d)$ be the join of $\Lambda(X ; \bar{d})$ and $(\Lambda(a, X) \otimes \Lambda Y, \delta)$ as in the diagram

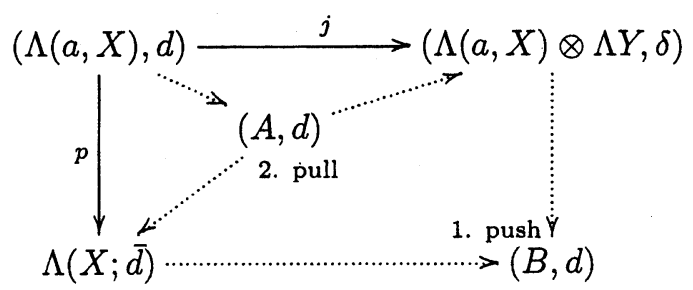

Thus, $(\Lambda X \otimes \Lambda Y, \bar{\delta}):=(\Lambda X, \bar{d}) \otimes_{(\Lambda(a, X), d)}(\Lambda(a, X) \otimes \Lambda Y, \delta)=(B, d)$ is a model of the pull back of $p$ and $j$, and

$$
(A, d)=\left(\Lambda X \oplus_{\Lambda X \otimes \Lambda Y} \Lambda(a, X) \otimes \Lambda Y, \bar{d}+\delta\right),
$$

which is isomorphic to the differential subspace $(\Lambda X \oplus(a \Lambda(a, X) \otimes \Lambda Y), \delta)$ of $(\Lambda(a, X) \otimes \Lambda Y, \delta)$. On the other hand,

$$
\left(\Lambda(a, X) / a \Lambda^{>k}(a, X), D\right)=\left(\Lambda X \oplus a\left(\Lambda(a, X) / \Lambda^{>k}(a, X)\right), D\right)
$$


is a differential subspace of $\left(\Lambda(a, X) / \Lambda^{>k}(a, X), D\right)$. Now, the surjective quasiisomorphism $\varphi$ in diagram (1) above induces a surjective map of differential algebras

$$
\begin{aligned}
\psi & :=\left(\operatorname{id}_{\Lambda X} \oplus a \varphi\right):(\Lambda X \oplus(a \Lambda(a, X) \otimes \Lambda Y), \delta) \\
& \rightarrow\left(\Lambda X \oplus a\left(\Lambda(a, X) / \Lambda^{>k}(a, X)\right), D\right)
\end{aligned}
$$

whose kernel isomorphic to $a \cdot \operatorname{ker} \varphi$, so that $H^{*}(\operatorname{ker} \psi)=a H^{*}(\operatorname{ker} \varphi)=0$. Thus, $\psi$ is a quasi-isomorphism. Since the functor $\mathcal{A}_{P L}$ and the geometric realization functor interchange homotopy pushouts and homotopy pullbacks, $\left(\Lambda(a, X) / a \Lambda^{>k}(a, X), D\right)$ is indeed a model of $F \bowtie_{E} W_{k}$.

To proceed, we retain the notation introduced in Lemma 4, and as usual, identify $x$ with $q(x)$ for all $x \in X$. Then, we can see that the condition on the differential $d$ is equivalent to $D X \subset \Lambda X$ in $\left(\Lambda(a, X) / \Lambda^{>k}(a, X), D\right)$. We next establish

Lemma 5. Let $\Lambda(a ; 0) \rightarrow \Lambda(a, V ; d) \stackrel{p}{\rightarrow} \Lambda(V ; \bar{d})$ be any model of $\xi$. There exists a minimal model $(\Lambda(a, X), d)$ of $E$ with

$$
d: X \rightarrow \Lambda X \oplus \Lambda^{+} a \cdot \Lambda^{>k}(a, X)
$$

iff there exists a map $\sigma$ in the diagram

$$
\Lambda(V ; \bar{d}) \underset{[}{\stackrel{\sigma}{\longrightarrow}\left(\Lambda(a, V) / a \Lambda^{>k}(a, V), D\right) \stackrel{\tilde{p}}{\longrightarrow}} \Lambda(V ; \bar{d})
$$

making it commute up to homotopy. (Here, $\tilde{p}$ is the map induced by p.)

Proof. Suppose the condition on $d$ is satisfied. By the remark preceding Lemma 5 , if we define $\sigma(x)=q(x), \sigma$ will be a map of differential algebras making the diagram above (with $V=X$ ) commute exactly.

Conversely, suppose we have such a $\sigma$. Because $\tilde{p}$ is surjective and $\Lambda(V ; \bar{d})$ is minimal, we may suppose without loss of generality (by Lemma 3 ) that $\tilde{p} \sigma=\operatorname{id}_{\Lambda V}$. We will use $\sigma$ to make a change of basis as follows. Our assumptions imply that there is a subspace $X \subset \Lambda(a, V)$ such that

$$
q: X \rightarrow \sigma(V)
$$

and

$$
\tilde{p} q: X \rightarrow V
$$

are isomorphisms. This implies that $\Lambda(a, X)=\Lambda(a, V)$, and so

$$
\left(\Lambda(a, V) / \Lambda^{>k}(a, V), D\right)=\left(\Lambda(a, X) / \Lambda^{>k}(a, X), D\right) .
$$

But then,

$$
D X=D \sigma(V)=\rho \bar{d}(V) \subset \sigma(\Lambda V)=\Lambda \sigma(V)=\Lambda X,
$$

so we are done.

We now relate $F \bowtie_{E} W_{k}$ and $F \bowtie_{E} G_{k} E$. As before, let $g_{k}: G_{k} E \rightarrow E$ denote the $k^{t h}$ Ganea fibration over $E$. 
Lemma 6. There exists a retraction $r$ in the diagram

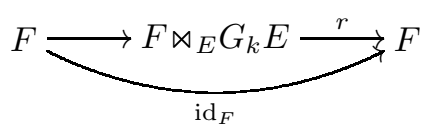

making it commute up to homotopy iff there exists a retraction $s$ in the diagram

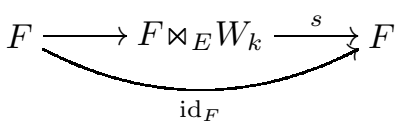

rendering it homotopy commutative.

Proof. Denote by $w_{k}: W_{k} \rightarrow E$ a geometric realization of the morphism $\Lambda(a, X) \rightarrow$ $\Lambda(a, X) / \Lambda^{>k}(a, X)$ and by $j$ (resp. by $\bar{j}$ ) the inclusion of $F$ in $F \bowtie_{E} G_{k} E$ (resp. $\left.F \bowtie_{E} W_{k}\right)$. Recall [ST] that there exist maps $\beta: G_{k} E \rightarrow W_{k}$ and $\alpha: W_{k} \rightarrow G_{k} E$ such that $g_{k} \circ \alpha \simeq w_{k}$ and $w_{k} \circ \beta \simeq g_{k}$. These induce maps $\bar{\alpha}: F \bowtie_{E} G_{k} E \rightarrow F \bowtie_{E} W_{k}$ and $\bar{\beta}: F \bowtie_{E} W_{k} \rightarrow G_{k} E$ such that $\bar{j} \circ \bar{\alpha} \simeq j$ and $j \circ \bar{\alpha} \simeq \bar{j}$. Thus, $j$ has a homotopy retraction if and only if $\bar{j}$ does.

Proof of Proposition 2. This is immediate from Lemmas 4, 5 and 6.

\section{§3. Proof of the theorem}

With the results and notation of the previous section, the second point of Theorem 1 now follows directly from

Proposition 7. Suppose the degree of a is even, $\Lambda(a ; 0) \rightarrow \Lambda(a, X ; d) \rightarrow \Lambda(X ; \bar{d})$ is a minimal $K-S$ extension and that $H^{*} \Lambda(X ; \bar{d})$ is a Poincaré duality algebra. Then, if $k \geq 0$ and $d: X \rightarrow \Lambda X \oplus \Lambda^{+} a \cdot \Lambda^{>k}(a, X)$, we have

$$
\operatorname{cat}_{0} \Lambda(a, X ; d) \geq \operatorname{cat}_{0} \Lambda(X ; \bar{d})+k .
$$

Proof. Though this closely follows the proof of [GJ, Thm. 1], we present it here for the sake of completeness.

Since the fibre is a Poincaré duality space and the base is Gorenstein, by [FHT2. Thm. 4.3], the total space is also Gorenstein. Moreover, since we may assume that $\operatorname{cat}_{0} \Lambda(a, X ; d)<\infty$, by Theorem 3.1 of the same article, we conclude that $H^{*} \Lambda(a, X ; \bar{d})$ is a Poincaré duality algebra. Then, by Propositions 5.1 and 5.3 , again of [FHT2], the formal dimension of the fibre is strictly greater than that of the total space.

Now note that we can write $d=\bar{d}+\sum_{i} a^{i} \eta_{i}$, where each $\eta_{i}$ is a derivation of $\Lambda X$ of odd degree. The assumption $d: X \rightarrow \Lambda X \oplus \Lambda^{+} a \cdot \Lambda^{>k}(a, X)$ then implies that $\eta_{i}: \Lambda^{p} X \rightarrow \Lambda^{\geq \max \{p-1, p+k+1-i\}} X$. In particular, if $\beta \in \Lambda^{\geq p} X$ satisfies $\bar{d} \beta=0$, then $d \beta=a \gamma$, where $\gamma \in \Lambda \geq p+k(a, X)$.

Denote $e_{0} \Lambda(X ; \bar{d})=e$, and let $\beta \in \Lambda^{\geq e} X$ be a cycle of degree equal to the formal dimension, representing the longest non-zero class. Since $\bar{d} \beta=0, d \beta=a \alpha$, where $d \alpha=0$ and $\alpha \in \Lambda^{\geq e+k}(a, X)$. Suppose that $\alpha$ is exact in $\Lambda(a, X ; d)$, say $\alpha=d \gamma$. Then, $\beta-a \gamma$ is a $d$-cycle of degree greater than the formal dimension of $\Lambda(a, X ; d)$, so $\beta-a \gamma=d z$ for some $z=z_{0}+a z_{1}$, where $z_{0} \in \Lambda X$. Comparison of coefficients of powers of $a$ on both sides of $\beta-a \gamma=d z$ yields $\bar{d} z_{0}=\beta$, which is a contradiction. Thus, the class of $\alpha$ is nonzero in $H^{*} \Lambda(a, X ; d)$, and so $e_{0} \Lambda(a, X ; d) \geq e+k$. 
It is hoped that one can eventually remove the assumption of Poincaré duality in the above. For the moment however, we content ourselves by remarking that Theorem 1 is not valid for a fibration over an odd Eilenberg-Mac Lane space (i.e. an odd rational sphere)

$$
F \rightarrow E \rightarrow \mathbf{S}_{\mathbf{Q}}^{2 n+1}
$$

Consider the fibration $\Lambda(u ; 0) \rightarrow \Lambda(u, v, w, x, y ; d) \rightarrow \Lambda(v, w, x, y ; 0)$ where all generators are of odd degree and the only non-zero differential is $d y=u v w x$. This satisfies all the hypotheses of Theorem 2 with $k=2$ except for the parity, but

$$
\operatorname{cat}_{0} \Lambda(u, v, w, x, y ; d)=5<6=\operatorname{cat}_{0} \Lambda(v, w, x, y ; 0)+2 .
$$

We now proceed with the proof of the first part of Theorem 1. Let $(M, A)$ be an NDR pair, denote by $i$ the inclusion $A \hookrightarrow M$, and as before let $g_{k}: G_{k} M \rightarrow M$ denote the $k^{t h}$ Ganea fibration over $M$. Recall that for a map $f: S \rightarrow M$, cat $f \leq n$ iff $f$ factors through $g_{n}$ iff there is an open cover $\left(S_{l}\right)_{1 \leq l \leq n+1}$ of $S$ such that for each $l$, the composition $S_{l} \hookrightarrow S \stackrel{f}{\rightarrow} M$ is homotopic to the constant map.

The first part of Theorem 1 now follows immediately from

Proposition 8. Suppose $F \rightarrow E \rightarrow K(\mathbf{Q}, 2 n)$ is a fibration with model $\Lambda(a ; 0) \rightarrow$ $\Lambda(a, X ; d) \rightarrow \Lambda(X ; \bar{d})$. If $k \geq 0$ and $d: X \rightarrow \Lambda X \oplus \Lambda^{+} a \cdot \Lambda^{>k}(a, X)$, then

$$
\operatorname{cat}_{0} E \leq \operatorname{cat}_{0}(E, F)+\max \left(\operatorname{cat}_{0} F, k+1\right)-k-1 .
$$

Proof. Suppose that $\operatorname{cat}(E, F)=n+1$. By $[\mathrm{M}], E$ is dominated by $F \bowtie_{E} G_{n} E$ and so $E$ is dominated by $F \bowtie_{E} G_{n} E \simeq F \bowtie_{E} G_{k} E \bowtie_{E} G_{n-k-1} E$ [C2]. Using the map $\beta$ used in the proof of Lemma 6 , we also see that $E$ is dominated by $F \bowtie_{E} W_{k} \bowtie_{E} G_{n-k-1} E$. Hence,

$$
\begin{aligned}
\operatorname{cat} E & \leq \operatorname{cat}\left(F \bowtie_{E} W_{k} \bowtie_{E} G_{n-k-1} E\right) \\
& \leq \operatorname{cat}\left(F \bowtie_{E} W_{k}\right)+\operatorname{cat} G_{n-k-1} E+1 \\
& \leq \operatorname{cat}\left(F \bowtie_{E} W_{k}\right)+n-k .
\end{aligned}
$$

The above inequalities also apply to the Q-localizations of all the spaces, so we may replace cat by cat $_{0}$. Now suppose that cat $_{0} F=m$, so that $\Lambda(X ; \bar{d})$ is a homotopy retract of $\Lambda X / \Lambda^{>m} X$. The condition on the differential implies that $A:=\left(\Lambda X \oplus a \cdot\left(\Lambda(a, X) / \Lambda^{>k}(a, X)\right), D\right)$ is a homotopy retract of

$$
B:=\left(\Lambda X / \Lambda^{>m} X \oplus a \cdot\left(\Lambda(a, X) / \Lambda^{>k}(a, X)\right), D\right) .
$$

Since the nilpotency of $B$ is at $\operatorname{most} \max (m, k+1)$, its rational category also has the same upper bound [FH]. Moreover, by Lemma $4, A$ is a model of $F \bowtie_{E} W_{k}$, so we obtain $\operatorname{cat}_{0}\left(F \bowtie_{E} W_{k}\right) \leq \max \left(\operatorname{cat}_{0} F, k+1\right)$, which completes the proof.

Note that this proof does not use the relativity of the homotopy in the definition of $\operatorname{cat}(E, F)$.

\section{$\S 4$. EXAMPLES}

Example 1. Consider the rational fibration $\left(\mathbf{C P}^{2}\right)^{3} \times\left(\mathbf{S}^{7}\right)^{2} \rightarrow E \rightarrow \mathbf{C P}^{\infty}$ with model

$$
(\Lambda a, 0) \rightarrow(\Lambda(a, b, c, e, u, v, w, x, y), d) \rightarrow(\Lambda(b, c, e, u, v, w, x, y), \bar{d}),
$$

where $a, b, c$ and $e$ are cycles of degree $2, d u=a^{4}, d v=b^{3}, d w=c^{3}, d x=e^{3}$, and $d y=a b c e$. The fibration is 2-trivial (while even the 0 -holonomy does not 
vanish), so the second point of Theorem 1 implies that cat ${ }_{0} E \geq 10$. The best lower bound that the Mapping Theorem or [C1] can provide is 8, while [J] and [GJ] can both be massaged to yield 9. In fact, if we apply [FH, lemma 6.6] to the fibration $\mathbf{S}^{7} \rightarrow E \rightarrow \mathbf{C P}^{3} \times\left(\mathbf{C P}^{2}\right)^{3}$, we obtain cat ${ }_{0} E \leq 10$, so indeed cat $_{0} E=10$ and the lower bound of Theorem 1 is sharp here.

Theorem 1 applied to (3) tells us that $\operatorname{cat}_{0}\left(E,\left(\mathbf{C P}^{2}\right)^{3} \times\left(\mathbf{S}^{7}\right)^{2}\right) \geq 5$.

Example 2. Consider the rational fibration $\mathbf{S}^{7} \times \mathbf{C P}^{2} \rightarrow E \rightarrow \mathbf{C P}^{\infty}$ with model $(\Lambda a, 0) \rightarrow(\Lambda(a, b, x, y), d) \rightarrow(\Lambda(b, x, y), \bar{d})$ where $a$ and $b$ are cycles of degree 2 and $d x=b^{3}$ and $d y=a^{4}+b^{2} a^{2}$. Again, the fibration is 2-trivial and so Theorem 1 gives cat $_{0} E \geq 5$. In fact, cat $_{0} E=5$, because a simple length-degree argument shows that $=\mathrm{e}_{0} E=5$. Theorem 1 implies also that $\operatorname{cat}_{0}\left(E, \mathbf{S}^{7} \times \mathbf{C P}^{2}\right) \geq 5$.

Example 3 . Consider the rational fibration $\mathbf{S}^{2} \times \mathbf{S}^{7} \times \mathbf{S}^{7} \rightarrow E \rightarrow \mathbf{C P}^{\infty}$ with Sullivan model given by $(\Lambda b, 0) \rightarrow(\Lambda(a, b, x, y), d) \rightarrow(\Lambda(a, x, y), \bar{d})$ where $|a|=$ $|b|=2,|x|=3,|y|=|z|=7, d a=d b=0, d x=a^{2}, d y=b^{4}$ and $d z=a^{3} b$. Theorem 1 implies that cat $_{0} E \geq 5$ and that $\operatorname{cat}_{0}\left(E, S^{2} \times S^{2} \times S^{5}\right) \geq 2$. If we apply [C2] to the fibration $S^{5} \rightarrow E \rightarrow C P(2) \times S^{2}$ with model $(\Lambda b, a, x, y, d) \rightarrow$ $(\Lambda(a, b, x, y), d) \rightarrow(\Lambda z, 0)$, we get $\operatorname{cat}_{0} E \leq 5$.

The authors would like to thank Yves Félix for many helpful discussions. The paper was also much improved as a result of the referee's comments, and we thank the referee for his/her help.

\section{REFERENCES}

[C1] Maxence Cuvilliez, Properties of semi-trivial fibrations, preprint of the Centre de Recerca matematica (Barcelona) 468 (2001).

[C2] L L $L$-S category of the total space in a fibration and $k$-monomorphisms, Topology and its Applications 109 (2001), 127-146. MR 2001m:55008

[CFJP] Maxence Cuvilliez, Yves Félix, Barry Jessup \& Paul-Eugène Parent, Rational LusternikSchnirelmann category of fibrations, to appear in J.P.A.A.

[FH] Y. Félix and S. Halperin, Rational L.-S. category and its applications, Trans. Amer. Math. Soc. 237 (1982), 1-37. MR 84h:55011

[FHL] Y. Félix, S. Halperin and Jean-Michel Lemaire, The rational LS category of products and Poincaŕe duality complexes, Topology 37, No. 4 (1998), 749-756. MR 99a:55003

[FHT1] Yves Félix, Stephen Halperin and Jean-Claude Thomas, Rational Homotopy Theory, Springer-Verlag, GTM. MR 2002d:55014

[FHT2] Gorenstein Spaces, Advances in Mathematics $\mathbf{7 1}$ (1) (1988), 92-112. MR 89k:55019

[GJ] Sonia Ghorbal and Barry Jessup, Estimating the rational LS-category of elliptic spaces, Proc. Amer. Math. Soc. 129 (2001), 1833-1842. MR 2001k:55032

[FaHu] E. Fadell, S. Y.Husseini, Relative category, product and coproducts, Rend. Sem. Mat. Fis. Milano 64 (1994), 99-115. MR 97f:55003

[I] N. Iwase, Ganea's conjecture on Lusternik-Schnirelmann category, Bull. London Math. Soc. 30 (1998), 623-634. MR 99j:55003

[J] Barry Jessup, Holonomy-Nilpotent Fibrations and Rational Lusternik-Schnirelmann Category, Topology 34 (4) (1995), 759-770. MR 96k:55014

[JS] I.M. James and W. Singhoff, On the category of fibre bundles, Lie groups and Froebenius maps, Contemp. Math. 227 (1996), 177-189. MR 2000a:55007

[M] Pierre-Marie Moyaux, Lower bounds for relative Lusternik-Schnirelmann Category, Manuscripta Math. 101 (2000), 533-542. MR 2001f:55006

[S] D. Sullivan, Infinitesimal computations in topology, Inst. Hautes Études Sci. Publ. Math. 47 (1978), 269-331. MR 58:31119 
[ST] Hans Scheerer and Daniel Tanré, Fibrations à la Ganea (French), Bull. Belg. Math. Soc. 4 (3) (1997), 333-353. MR 98g:55003

[T] G.H. Toomer, Lusternik-Schnirelmann category and the Moore spectral sequence, Math. Z. 138 (1974), 123-143. MR 50:8509

Centre de Recerca Matemàtica, Barcelona, Spain

E-mail address: mcuvilli@crm.es

Department of Mathematics and Statistics, University of Ottawa, Ottawa, Ontario, CANADA K1N 6N5

E-mail address: bjessup@uottawa.ca 